\title{
Avaliação da satisfação quanto ao jogo educativo NeuroGame-Card como estratégia de ensino em Enfermagem
}

\author{
Evaluation of satisfaction regarding the NeuroGame-Card educational game as a Nursing teaching \\ strategy \\ Evaluación de la satisfacción con respecto al juego educativo NeuroGame-Card como estrategia de \\ enseñanza de Enfermería
}

Lóren Maria da Cruz Rodrigues ORCID: https://orcid.org/0000-0002-6835-5700 Universidade Federal de Uberlândia, Brasil E-mail: lorenmariacruz@gmail.com

Raphael Silva Rodrigues ORCID: https://orcid.org/0000-0002-8892-1108 Universidade Federal de Uberlândia, Brasil E-mail: rapha_silva14@hotmail.com

Suely Amorim de Araújo ORCID: https://orcid.org/0000-0001-9234-166X Universidade Federal de Uberlândia, Brasil E-mail: suamorim1@yahoo.com.br

Clesnan Mendes-Rodrigues

ORCID: https://orcid.org/0000-0002-8871-7422 Universidade Federal de Uberlândia, Brasil E-mail: clesnan@bol.com.br

Valéria Nasser Figueiredo

ORCID: https://orcid.org/0000-0001-5793-112X Universidade Federal de Uberlândia, Brasil E-mail: valeria.n.figueiredo@gmail.com

Patrícia Costa dos Santos da Silva ORCID: https://orcid.org/0000-0001-9643-1865 Universidade Federal de Uberlândia, Brasil E-mail: patriciacostaunifenas@ hotmail.com

\begin{abstract}
Resumo
O objetivo deste estudo foi avaliar a satisfação dos estudantes de Enfermagem quanto ao jogo NeuroGame-Card que enfoca uma estratégia de ensino dos doze pares de nervos cranianos. Trata-se de um estudo analítico-descritivo, de abordagem quantitativa, desenvolvido com uma amostra de 44 participantes/estudantes/jogadores. Para a coleta de dados, que ocorreu no período de outubro a dezembro de 2018, todos os estudantes realizaram uma partida do jogo e, logo em seguida, utilizou-se a escala EGameFlow para a avaliação da satisfação, juntamente com um instrumento de perfil e de afinidade por jogos, após a aprovação do Comitê de Ética em Pesquisa. Todos os estudantes (100\%) achavam que os jogos ajudam no aprendizado e que deveriam ser utilizados como estratégia de ensino. Quanto aos resultados, constatou-se que $86,4 \%$ dos jogadores têm afinidade por jogos e 79,5\% possuem afinidade por jogos em equipe. Já em relação à escala EGameFlow, no domínio clareza dos objetivos, a média foi 6,43 e, no domínio melhoria do conhecimento, a média foi 6,50, com os demais domínios maior ou igual a 5,19. O perfil dos estudantes, sua afinidade por jogos individuais ou em grupo e sua afinidade por determinado tipo de jogo foram capazes de predizer os domínios do EGameFlow;. Com isso, devem ser utilizadas como estratégias de construção de futuros jogos. Conclui-se que o nível geral de satisfação dos estudantes com o jogo NeuroGame-Card foi positivo como estratégia educacional para o ensino do exame físico dos doze pares de nervos cranianos.
\end{abstract}

Palavras-chave: Ensino; Educação; Neurologia e enfermagem; Jogos.

\section{Abstract}

The objective of this study was to evaluate the satisfaction of nursing students regarding the neurogame-card game that focuses on a teaching strategy of the twelve cranial nerve pairs. This is an analytical-descriptive study, with a quantitative approach, developed with a sample of 44 participants/students/players. For data collection, which occurred from October to December 2018, all students performed a game match and then the EGameFlow scale was used to assess satisfaction, together with a game profile and affinity instrument, after the approval of the Research 
Ethics Committee. All students (100\%) they thought that games help with learning and should be used as a teaching strategy. As for the results, it was found that $86.4 \%$ of the players have affinity for games and $79.5 \%$ have affinity for team games. In relation to the EGameFlow scale, in the domain clarity of objectives, the average was 6.43 and, in the domain improvement of knowledge, the average was 6.50, with the other domains greater than or equal to 5.19. The students' profile, their affinity for individual or group games, and their affinity for a particular type of game were able to predict EGameFlow's domains; With this, they should be used as strategies for building future games. It was concluded that the general level of student satisfaction with the NeuroGame-Card game was positive as an educational strategy for teaching the physical examination of the twelve pairs of cranial nerves.

Keywords: Teaching; Education; Neurology; Nursing; Gaming.

\section{Resumen}

El objetivo de este estudio era evaluar la satisfacción de los estudiantes de enfermería con respecto al juego de cartas neurojuegos que se centra en una estrategia de enseñanza de los doce pares nerviosos craneales. Se trata de un estudio analítico-descriptivo, con un enfoque cuantitativo, desarrollado con una muestra de 44 participantes/estudiantes/jugadores. Para la recopilación de datos, que se produjo de octubre a diciembre de 2018 , todos los estudiantes realizaron una coincidencia de juego y luego la escala EGameFlow se utilizó para evaluar la satisfacción, junto con un perfil de juego y un instrumento de afinidad, después de la aprobación del Comité de Ética de Investigación. Todos los estudiantes (100\%) pensaron que los juegos ayudan con el aprendizaje y deben ser utilizados como una estrategia de enseñanza. En cuanto a los resultados, se encontró que el 86,4\% de los jugadores tienen afinidad por los juegos y el 79,5\% tienen afinidad por los partidos de equipo. En relación con la escala EGameFlow, en la claridad de los objetivos del dominio, la media fue de 6,43 y, en la mejora del dominio del conocimiento, la media fue de 6,50, con los demás dominios superiores o iguales a 5,19. El perfil de los estudiantes, su afinidad por los juegos individuales o grupales, y su afinidad por un tipo de juego en particular fueron capaces de predecir los dominios de EGameFlow; Con esto, deben ser utilizados como estrategias para construir juegos futuros. Se concluyó que el nivel general de satisfacción del estudiante con el juego NeuroGame-Card era positivo como una estrategia educativa para enseñar el examen físico de los doce pares de nervios craneales.

Palabras clave: Enseñanza; Educación; Neurología; Enfermería; Juego.

\section{Introdução}

Com os avanços tecnológicos e científicos, ferramentas e metodologias de ensino são disponibilizadas para proporcionar o aprendizado aos estudantes. Assim, os jogos têm obtido relevância na área de saúde, pois representam um recurso educacional criativo e de participação coletiva (Olympio \& Alvim, 2018). Dessa forma, diversos jogos foram desenvolvidos e são utilizados em diferentes cursos e disciplinas (Maddineshat, et al., 2018; Thornton Bacon, et al., 2018; Nasiri, et al., 2019; dos Santos, et al., 2020; Mitchell, et al., 2021; Tyerman, et al., 2021). Nesse sentido, a inclusão de jogos nos cursos da área de saúde tem ganhado espaço, por ser uma metodologia que aguça a criatividade, promove a participação ativa e em grupo no processo de ensino-aprendizagem, além de estimular o raciocínio clínico (Holanda, et al., 2012; GómezUrquiza, et al., 2019; dos Santos, et al., 2020; Mitchell, et al., 2021; Tyerman, et al., 2021).

$\mathrm{Na}$ área de Enfermagem, a utilização de jogos tem o potencial de envolver os estudantes, aprimorar o aprendizado, promover a participação ativa, facilitar a aquisição e a compreensão de conteúdos complexos. Além disso, permite a interação entre professor e aluno (Gallegos, et al., 2017; Castro, et al., 2019; Mitchell, et al., 2021). No ensino da Enfermagem, a propedêutica neurológica é vista como um assunto complexo e bastante árduo. A literatura aponta fragilidades no saber dos enfermeiros em relação à avaliação neurológica (Da Paixão Oliveira, et al., 2016). Nesse sentido, estudos que possam trazer estratégias de ensino para melhorar a avaliação do estado neurológico são bem-vindas, sobretudo no que diz respeito à avaliação dos pares de nervos cranianos que é extremamente relevante na prática clínica.

$\mathrm{Na}$ atualidade, a formação do enfermeiro transcende a atualização científica, pedagógica e didática, amplificando os espaços para a criatividade, para a interação e para o diálogo. Assim, ressalta-se a necessidade de desenvolver novos modelos de ensino-aprendizagem para a avaliação dos pares de nervos cranianos voltados aos profissionais e aos acadêmicos de Enfermagem, capacitando-os no desempenho de ações de detecção precoce e de acompanhamento das disfunções neurológicas. Nessa perspectiva, a utilização e a adoção do jogo podem ser consideradas uma alternativa bastante promissora (Holanda, et al., 2012; Dias et al., 2016). No entanto, há uma escassez de evidências científicas que investiguem a satisfação 
dos estudantes com a utilização do jogo para a avaliação dos pares de nervos cranianos, com a finalidade de promover experiências interdisciplinares. O jogo NeuroGame-Card que enfoca os doze pares de nervos cranianos já foi elaborado e teve sua avaliação de conteúdo (Rodrigues, et al., 2020), mas sua validação na prática e a satisfação dos estudantes são desconhecidas.

Assim, o objetivo deste estudo foi avaliar o grau de satisfação dos estudantes de um curso de Graduação em Enfermagem, quanto ao uso do jogo NeuroGame-Card como estratégia de ensino que enfoca os doze pares de nervos cranianos.

\section{Metodologia}

Trata-se de um estudo analítico-descritivo e quantitativo, realizado no Curso de Graduação em Enfermagem da Faculdade de Medicina da Universidade Federal de Uberlândia (FAMED-UFU), Minas Gerais, Brasil. A população do estudo foi composta pelos alunos a partir do quarto período do curso de graduação em Enfermagem, pois, nesses períodos, os estudantes já possuem conhecimento prévio obtido na disciplina Sistematização Assistencial de Enfermagem (SAE) que ocorre no terceiro semestre e trata da avaliação dos doze pares de nervos cranianos. O recrutamento dos alunos para participarem do estudo foi feito por intermédio de convite verbal nas salas de aula. A coleta se deu após terem sido orientados quanto aos objetivos e à proposta da pesquisa e assinado o termo de consentimento livre e esclarecido. $\mathrm{O}$ estudo foi aprovado pelo Comitê de Ética em Pesquisa da Universidade Federal de Uberlândia, sob o número do parecer 2.294.871.

O tamanho amostral total foi de 44 graduandos do Curso de Graduação em Enfermagem da UFU. O tamanho da amostra foi obtido com base na conveniência populacional. Esse número total foi estipulado por se tratar de uma amostra não probabilística, baseada na experiência dos pesquisadores no campo de pesquisa, fundamentada em raciocínios instruídos por conhecimentos teóricos (Oliveira, 2001). Como critérios de inclusão do presente estudo, estabeleceu-se a faixa etária igual ou maior de 18 anos de idade de alunos que estavam matriculados no curso de graduação em Enfermagem da UFU e que já tivessem cursado a disciplina de SAE, ministrada no terceiro período de graduação em Enfermagem. Não houve critérios de exclusão.

Para que os alunos realizassem o estudo prévio ou a revisão sobre o exame físico dos doze pares de nervos cranianos, disponibilizou-se, com antecedência de aproximadamente 20 dias da aplicação do jogo, por e-mail, referencial teórico sobre a avaliação dos 12 pares de nervos cranianos. $\mathrm{O}$ acesso ou a leitura desse material não foram avaliados. Utilizou-se o jogo NeuroGame-Card, elaborado por Rodrigues, et al., (2020) e aplicado nas salas de aula localizadas no Bloco 8C da Universidade, no período de outubro a dezembro de 2018. Logo após o jogo, realizou-se a aplicação dos instrumentos e da escala pelos pesquisadores do estudo. Todos os participantes realizaram somente uma partida do jogo.

Para a coleta de dados, utilizou-se um instrumento composto por duas partes. A primeira parte foi um instrumento elaborado pelos próprios pesquisadores do estudo, sendo constituída pelas seguintes questões do perfil como sexo, idade, período do curso, além de questões sobre a afinidade. Avaliou-se se os jogadores tinham afinidade por jogos do tipo tabuleiro, online, simulador, cartas, memória e de memória. A escala de afinidade por jogos baseou-se em uma escala do tipo Likert, variando de um a cinco (1 - Nenhuma afinidade, 2 - Pouca afinidade, 3 - Indiferente, 4 - Afinidade, 5 - Muita afinidade). A segunda parte constituiu-se de uma escala validada, denominada EGameFlow, elaborada por Fu, et al., (2009) e traduzida para a língua portuguesa por Tsuda, et al., (2014), a qual avalia a satisfação dos alunos com relação ao jogo educacional, auxilia na compreensão dos pontos fortes e pontos fracos do jogo do ponto de vista dos jogadores. Essa escala possui oito dimensões, sendo estas concentração, desafios, autonomia, clareza dos objetivos, feedback, imersão, interação social e melhoria do conhecimento. Cada dimensão apresenta diversos itens que foram avaliados pelos alunos, os quais atribuíram 
uma nota de um a sete, sendo que um é considerado "fraco" e o sete é considerado "forte". A nota final da avaliação de cada dimensão do jogo é obtida a partir da média das notas dos itens que a compõem (Fu, et al., 2009).

Todas as variáveis qualitativas foram apresentadas como frequência absoluta e relativa. Já as variáveis quantitativas, com média, mediana, desvio padrão, mínimo e máximo. Dos 44 respondentes, em 9 casos, o perfil e as escalas não puderam ser pareadas por ausência de identificação dos respondentes. As variáveis "Você acha que os jogos ajudam no aprendizado" e "Você acha que os jogos podem ser utilizados no ensino da enfermagem" foram excluídas de algumas análises, uma vez que 100\% dos alunos responderam $\operatorname{Sim}(\mathrm{n}=44$ alunos). As escalas de afinidade individualmente e a escala média de afinidade foram correlacionadas entre si. Foram correlacionadas também com os domínios da escala pelo coeficiente de Correlação de Spearman, cuja significância foi testada com o teste $t$ de Student. Para todas as análises, adotou-se significância de 5\%.

Os dados de frequência de estudo foram dicotomizados em se diariamente (atribuído sim) e em outras frequências (atribuído não). Já a modalidade de jogos, foi dicotomizada em se tem afinidade por jogos em grupo (atribuído sim) e individual (atribuído não). Todas as variáveis dicotômicas sim ou não, foram recodificadas em 1 e 0 , respectivamente. Para as escalas de afinidade a diferentes tipos de jogos, foi calculado como uma nova variável a afinidade média por jogos através da média dos sete itens de afinidade (podendo oscilar de um a cinco). Os 42 itens da escala foram calculados, obtendo-se a média para cada um dos oito domínios da escala, como proposto pelo artigo de validação (Tsuda, et al., 2014).

Em relação à capacidade de predição do perfil e de as escalas de afinidades predizerem os domínios da escala EGameFlow, a qual pode apresentar dimensões multifatoriais, utilizou-se a regressão linear múltipla, pelo método dos mínimos quadrados. Para a seleção das variáveis de importância na predição das dimensões do constructo avaliado, utilizou-se o método backward, baseando-se no critério de exclusão do modelo, a probabilidade maior que $10 \%$. Os coeficientes do modelo foram testados com teste $t$ de Student. Como os modelos não foram construídos com o intuito de predição e, sim, para descrever os indivíduos estudados nessa população, os pressupostos dos modelos e as métricas de qualidade de ajuste não foram testados.

\section{Resultados}

Houve um predomínio de mulheres $(90,90 \%)$ e de estudantes jovens com média de 22 anos cursando predominantemente o sexto e quarto período $(43,20 \%$ e $36,40 \%$, respectivamente). A maioria relata hábito de estudo $(90,90 \%)$, mas uma frequência de estudos semanal $(56,80 \%)$. Os participantes também relataram afinidade por jogos $(86,40 \%)$, preferencialmente na modalidade em equipe $(79,50 \%)$ (Tabela $1 ; 2)$ Todos os estudantes achavam que os jogos ajudam no aprendizado $(100 \%, n=44)$ e que podem ser utilizados no ensino da Enfermagem $(100 \%, n=44)$.

As escalas de afinidades por jogos mostraram médias e medianas próximas ou superiores a três (amplitude de 2,64 a 4,02), o que evidencia uma afinidade de média a alta para jogos. A escala de afinidade média foi de 3,56. Já os domínios da escala EGameFlow, tiveram sempre média acima de cinco, oscilando de 5,19 (Domínio Autonomia) a 6,50 (Domínio Melhoria do conhecimento). (Tabela 2) Esse achado reforça a afinidade pelos jogos e a compreensão, além do fato de que essa ferramenta didática colabora com o aprendizado em Enfermagem.

A afinidade por jogos foi correlacionada com o Domínio Clareza dos objetivos ( $r s=-0,457 ; p<0,05)$, evidenciando que quanto maior a afinidade por jogos menores foram os valores desse domínio. A afinidade por jogos de memória foi correlacionada com o Domínio Interação Social ( $r s=0,351 ; p<0,05)$, evidenciando que quanto maior, maior o valor obtido na outra escala. Os demais itens como o período, a idade e outros itens das escalas de afinidade, não foram correlacionados com os domínios da escala de avaliação. (Tabela 3)

Quando se avalia a capacidade de predição do perfil e da afinidade por jogos em relação aos dominios da escala EGameFlow, encontram-se resultados muito distintos para cada um dos domínios (Tabela 4). Pensando no domínio Melhoria 
do conhecimento, como um reflexo de o jogo ser uma estratégia adequada de ensino, percebe-se que ser mulher aumentou o domínio $(B i=0,84)$, juntamente com ter preferência pela modalidade em grupo $(B i=0,83)$, quanto maior a afinidade por jogos de tabuleiro $(B i=0,26)$; sendo que somente o aumento da afinidade por jogos diminuiu o domínio $(B i=-0,31)$. Eventualmente o trabalho em grupo, a construção de estratégias como a afinidade por jogos de tabuleiro estão associados à montagem de relações, de estratégias e maior correlação com a prática de raciocino clínico, uma vez que o aluno precisa conectar as informações numa sequência para chegar a uma conclusão clínica ou atingir um objetivo do jogo.

Tabela 1. Perfil dos alunos de Enfermagem que participaram da avaliação do jogo NeuroGame-Card. Uberlândia, MG, 2018.

\begin{tabular}{llc}
\hline Variável & Estrato & $\%(\mathrm{n})$ \\
\hline \multirow{2}{*}{ Sexo } & Feminino & $90,90(40)$ \\
& Masculino & $9,10(4)$ \\
\hline \multirow{2}{*}{ Período do curso } & Quarto & $36,40(16)$ \\
& Quinto & $4,50(2)$ \\
& Sexto & $43,20(6)$ \\
& Sétimo & $11,40(5)$ \\
& Nono & $4,50(2)$ \\
\hline \multirow{2}{*}{ Possui hábito de estudo } & Não & $90,90(40)$ \\
& Sim & $9,10(4)$ \\
\hline \multirow{2}{*}{ Com que frequência você estuda } & Diariamente & $31,80(14)$ \\
& Semanalmente & $56,80(25)$ \\
& Quinzenalmente & $9,10(4)$ \\
Com que frequência você estuda dicotomizado & Mensamente & $2,30(1)$ \\
\hline \multirow{2}{*}{ Possui afinidade por jogo } & Outros & $31,80(14)$ \\
\hline Com qual das modalidades de jogos abaixo você & Jogos Individuais & $68,20(30)$ \\
\hline possui maior afinidade & Jogos em equipe & $79,50(35)$ \\
\hline
\end{tabular}

Fonte: Dados da pesquisa. 
Tabela 2. Análise descritiva do perfil, das escalas de afinidade com jogos e dos domínios da escala EGameFlow na avaliação do jogo NeuroGame-Card por estudantes de Enfermagem. Uberlândia, MG, 2018.

\begin{tabular}{lccccr}
\hline Variável & Média & Desvio Padrão & Mediana & Mínimo & Máximo \\
\hline Idade (anos) & 22,39 & 4,41 & 21,00 & 19,00 & 38 \\
Afinidade por jogos & 4,02 & 1,15 & 4,00 & 1,00 & 5 \\
Afinidade por jogos de tabuleiro & 3,89 & 1,24 & 4,00 & 1,00 & 5 \\
Afinidade por jogos online & 2,64 & 1,48 & 3,00 & 1,00 & 5 \\
Afinidade por jogos de simulador & 3,34 & 1,48 & 3,00 & 1,00 & 5 \\
Afinidade por jogos de cartas & 3,55 & 1,41 & 4,00 & 1,00 & 5 \\
Afinidade por jogos de memória & 3,57 & 1,37 & 4,00 & 1,00 & 5 \\
Afinidade por jogos de estratégia & 3,91 & 1,16 & 4,00 & 1,00 & 5 \\
Escala de Afinidade Média & 3,56 & 0,90 & 3,64 & 1,43 & 5 \\
Domínio Concentração & 5,46 & 0,80 & 5,38 & 3,75 & 7 \\
Domínio Desafio & 5,83 & 0,82 & 5,94 & 3,56 & 7 \\
Domínio Autonomia & 5,19 & 1,02 & 5,29 & 2,43 & 7 \\
Domínio Clareza dos objetivos & 6,43 & 0,79 & 6,67 & 3,00 & 7 \\
Domínio Feedback & 5,75 & 1,26 & 6,13 & 2,50 & 7 \\
Domínio Imersão & 5,95 & 1,17 & 6,13 & 2,50 & 7 \\
Domínio Interação Social & 5,79 & 1,21 & 6,00 & 1,67 & 7 \\
Domínio Melhoria do conhecimento & 6,51 & 0,72 & 6,80 & 3,60 & 7 \\
\hline
\end{tabular}

Fonte: Dados da pesquisa.

Tabela 3. Correlações de Spearman entre os domínios da escala EGameFlow e as variáveis do perfil e de afinidade com jogos dos estudantes de Enfermagem que jogaram o NeuroGame-Card. Uberlândia, MG, 2018.

\begin{tabular}{lcccccccccc}
\hline \multirow{2}{*}{ Domínio } & \multicolumn{10}{c}{ Variável ${ }^{1,2}$} \\
\cline { 2 - 10 } & 1 & 2 & 3 & 4 & 5 & 6 & 7 & 8 & 9 & 10 \\
\hline Concentração & $-0,332$ & $-0,035$ & $-0,042$ & 0,083 & 0,046 & 0,057 & 0,092 & 0,133 & 0,278 & 0,118 \\
Desafio & $-0,066$ & $-0,045$ & $-0,28$ & 0,145 & $-0,108$ & 0,005 & 0,084 & 0,17 & 0,086 & 0,017 \\
Autonomia & $-0,06$ & 0,044 & $-0,236$ & 0,073 & $-0,063$ & 0,007 & 0,007 & 0,169 & 0,103 & $-0,003$ \\
Clareza e & & & & & & & & & & \\
Objetividade & 0,187 & $-0,002$ & $-0,457 *$ & $-0,006$ & $-0,213$ & $-0,305$ & 0,084 & $-0,021$ & $-0,148$ & $-0,181$ \\
Feedback & $-0,102$ & $-0,031$ & $-0,025$ & 0,165 & 0,055 & 0,159 & $-0,039$ & 0,122 & $-0,081$ & 0,054 \\
Imersão & $-0,017$ & 0,055 & $-0,17$ & $-0,089$ & $-0,162$ & $-0,039$ & $-0,082$ & 0,221 & $-0,072$ & $-0,088$ \\
Interação Social & 0,062 & 0,274 & $-0,096$ & 0,032 & 0,138 & 0,191 & $-0,137$ & $0,351 *$ & 0,021 & 0,124 \\
Melhoria do & & & & & & & & & & \\
conhecimento & $-0,141$ & 0,029 & $-0,27$ & 0,197 & $-0,023$ & 0,034 & 0,014 & 0,066 & $-0,102$ & 0,014 \\
\hline
\end{tabular}

${ }^{1}$ Variável: 1 -Período, 2 -Idade (anos), 3 - Afinidade por jogos, 4 - Afinidade por jogos de tabuleiro, 5 - Afinidade por jogos online, 6 - Afinidade por jogos de simulador, 7 - Afinidade por jogos de cartas, 8 - Afinidade por jogos de memória, 9 - Afinidade por jogos de estratégia, 10 - Escala de Afinidade Média

${ }^{2}$ Valores seguidos de * são significativos a 5\%, pelo teste $t$ de Student.

Fonte: Dados da pesquisa. 
Tabela 4. Regressão linear múltipla para os domínios da escala de avaliação EGameFlow em função de algumas variáveis preditoras na avaliação do jogo NeuroGame-Card realizada por estudantes de Enfermagem. Uberlândia, MG, 2018.

\begin{tabular}{|c|c|c|c|c|c|}
\hline Domínio & Variável preditor & $B i$ & $E P$ & $t$ & $p$ \\
\hline \multirow{5}{*}{ Concentração } & Constante & 6,44 & 0,81 & 7,97 & $<0,001$ \\
\hline & Se prefere jogos em grupo & 0,60 & 0,34 & 1,78 & 0,086 \\
\hline & Período do curso & $-0,22$ & 0,10 & $-2,12$ & 0,042 \\
\hline & Escala de Afinidade por jogos & $-0,34$ & 0,14 & $-2,45$ & 0,020 \\
\hline & Escala de Afinidade por jogos de estratégia & 0,29 & 0,13 & 2,20 & 0,035 \\
\hline \multirow{3}{*}{ Desafio } & Constante & 8,36 & 1,11 & 7,55 & $<0,001$ \\
\hline & Idade & $-0,07$ & 0,03 & $-2,02$ & 0,051 \\
\hline & Escala de Afinidade por jogos & $-0,25$ & 0,13 & $-1,93$ & 0,062 \\
\hline \multirow{2}{*}{ Autonomia } & Constante & 3,68 & 0,47 & 7,77 & $<0,001$ \\
\hline & Se Sexo feminino & 1,63 & 0,50 & 3,24 & 0,003 \\
\hline \multirow{5}{*}{$\begin{array}{l}\text { Clareza e } \\
\text { Objetividade }\end{array}$} & Constante & 6,05 & 0,64 & 9,52 & $<0,001$ \\
\hline & Tem afinidade por jogos (sim) & 1,07 & 0,52 & 2,08 & 0,047 \\
\hline & Período do curso & 0,18 & 0,09 & 2,04 & 0,050 \\
\hline & Escala de Afinidade por jogos & $-0,68$ & 0,17 & $-4,12$ & $<0,001$ \\
\hline & Escala de Afinidade por jogos de cartas & 0,36 & 0,09 & 4,05 & $<0,001$ \\
\hline \multirow{4}{*}{ Feedback } & Constante & 5,43 & 0,56 & 9,69 & $<0,001$ \\
\hline & Se tem frequência de estudos diária & 0,83 & 0,44 & 1,89 & 0,069 \\
\hline & Tem afinidade por jogos (sim) & $-0,96$ & 0,54 & $-1,78$ & 0,085 \\
\hline & Se prefere jogos em grupo & 1,16 & 0,47 & 2,46 & 0,02 \\
\hline \multirow{2}{*}{ Imersão } & Constante & 4,81 & 0,58 & 8,35 & $<0,001$ \\
\hline & Se sexo feminino & 1,36 & 0,61 & 2,22 & 0,034 \\
\hline \multirow{3}{*}{ Interação Social } & Constante & 5,50 & 0,64 & 8,55 & $<0,001$ \\
\hline & Escala de Afinidade por jogos de memória & 0,53 & 0,18 & 3,03 & 0,005 \\
\hline & Escala de Afinidade por jogos de estratégia & $-0,41$ & 0,21 & $-2,01$ & 0,052 \\
\hline \multirow{6}{*}{$\begin{array}{l}\text { Melhoria do } \\
\text { conhecimento }\end{array}$} & Constante & 4,75 & 0,74 & 6,42 & $<0,001$ \\
\hline & Se sexo feminino & 0,84 & 0,34 & 2,44 & 0,021 \\
\hline & Escala de Afinidade por jogos de estratégia & 0,66 & 0,38 & 1,74 & 0,092 \\
\hline & Se prefere jogos em grupo & 0,83 & 0,27 & 3,13 & 0,004 \\
\hline & Escala de Afinidade por jogos & $-0,31$ & 0,11 & $-2,71$ & 0,011 \\
\hline & Escala de Afinidade por jogos de tabuleiro & 0,26 & 0,09 & 2,78 & 0,009 \\
\hline
\end{tabular}

Legenda: $B i$ : i-ésima estimativa dos parâmetros do modelo, $t$ : estatística do teste $t$ de Student para a estimativa dos coeficientes, $E P$ : erro padrão, $p$ : probabilidade

Fonte: Dados da pesquisa.

\section{Discussão}

O processo de design dos jogos educacionais deve seguir as etapas propostas na literatura científica: estabelecer os problemas; criar alternativas de design; selecionar a solução e avaliar o processo (Tsuda, et al., 2014). O jogo NeuroGame- 
Card já tinha passado pelas três primeiras etapas iniciais (Rodrigues, et al., 2020). Neste estudo, realizou-se a avaliação do jogo pelos estudantes de Enfermagem com o intuito de coletar informações sobre a experiência dos participantes com a finalidade de aprimorar o design do jogo. Nota-se que o objetivo foi alcançado e que foi possível analisar a repercussão do NeuroGame-Card enquanto ferramenta de ensino, pois, nos oito domínios da escala EGameFlow, as médias foram superiores a seis pontos em dois domínios (clareza dos objetivos e melhoria do conhecimento). Já em seis domínios, as médias foram acima de cinco pontos (concentração, desafio, autonomia, feedback, imersão e interação social), o que mostra satisfação dos estudantes com a utilização do jogo como estratégia de ensino. De fato, salienta-se a importância do desenvolvimento e da avaliação de instrumentos pedagógicos que se alinhem com as transformações da atualidade nos cuidados de saúde e que ensinem os estudantes de Enfermagem um alto nível de habilidades de raciocínio clínico e de conhecimento baseado em evidências científicas (Johnsen, et al., 2018).

Quanto ao perfil sociodemográfico, as variáveis sexo e idade coincidem com a literatura por Hirsch, et al., (2014). Em relação ao hábito e à frequência de estudo, observou-se certa contradição, pois, apesar de mencionarem estudar semanalmente, relatam não ter o hábito de estudo. Tal achado pode ser justificado pelo estudo de Oliveira \& Oliveira (2007) o qual refere que os estudantes apresentavam o hábito de estudar apenas uma hora por dia, por motivos de trabalho e de outros afazeres, ou seja, muitas vezes, o estudante dedicava poucas horas ao estudo.

No que tange à afinidade por jogos, $86,4 \%$ dos participantes afirmam ter preferência por jogos em equipe. A afinidade por jogos tem como característica a promoção do processo ensino-aprendizagem, do ensino de princípios e dos conceitos da Análise Experimental do Comportamento para adultos e jovens, dentre outros. Panosso, et al., (2015) concluíram que os jogos educativos apresentam traços de estratégias de ensino e de motivação, sendo capazes de contribuir para que esse ensino seja eficaz e eficiente.

Neste estudo, a maioria dos estudantes de Enfermagem relata ter afinidade por jogos. A literatura científica mostra que o uso da tecnologia da informação pode auxiliar na construção de materiais elaborados, com tabuleiros, cartas e dados coloridos, os quais podem conquistar pelo visual atraente. A receptividade perante esse material visualmente interessante gera uma impressão agradável, capaz de despertar uma afinidade pelo conteúdo exposto (Favaretto, 2017). O jogo NeuroGame-card utilizou diversos estratégias visuais com a finalidade de criar um tabuleiro visualmente atraente, com cartas coloridas, de forma a promover afinidade dos estudantes com o jogo (Rodrigues, et al., 2020).

No que se refere ao item concentração, a média foi 5,46, sendo que os achados do presente estudo coadunam com estudo de Neves et al., (2014) o qual relata que, para ser divertido, o jogo deve conduzir o jogador a um estado de concentração. Dessa forma, o interesse é proporcional ao nível de concentração que a atividade estará exigindo do estudante. Neste estudo, os acadêmicos de Enfermagem demonstraram interesse. Evitaram-se distrações não relacionadas à tarefa central a ser completada, sendo que as perguntas efetuadas foram claras e nenhuma informação a mais foi fornecida ao jogador além daquilo que era necessário executar, o que pode ser avaliado de forma positiva por ajudá-lo a manter sua concentração. Além disso, a escassez de estímulos sonoros, em virtude de o NeuroGame-Card se tratar de um jogo de tabuleiro, foi uma característica importante para se manter a concentração.

O segundo domínio da escala EGameFlow é o desafio. Nesse item, a média foi 5,83, o que pode apontar para uma parcela de jogadores que se sentiram pouco desafiados. O NeuroGame-Card possui cartões com perguntas que, se não forem respondidas corretamente ou a ação não for executada pela equipe, deve retornar ao monte de cartas com a última carta a ser utilizada, ficando embaixo de todas as outras. Nessa situação, o mediador não mencionava a alternativa correta em caso de erro (Rodrigues, et al., 2020). Isso foi realizado, visando aproveitar melhor o jogo, de forma que os estudantes não se sentissem entediados, conforme o jogo alavancasse. No estudo de Tsuda, et al., (2014), o jogo "Armas e barões" obteve a nota cinco no domínio desafio, relatando que o jogo proporcionava uma experiência com dificuldade adequada, o que fez com que os 
participantes não se sentissem ansiosos ou mesmo entediados. Nesse jogo, o jogador vai aprendendo as melhores estratégias, ou seja, o aumento da dificuldade acontece ao longo do jogo. Neves, et al., (2014) referem que as habilidades do jogador e seu interesse dependem do aumento gradativo do desafio.

O domínio autonomia obteve a média mais baixa de todos os domínios, provavelmente por se tratar de um jogo de equipe e, com isso, o estudante tivesse a sensação de não estar controlando o jogo. Um estudo salienta que a autonomia do jogo expressa a experiência ds jogadores em se colocarem em uma postura de liberdade para a tomada de decisões e para as escolhas no jogo (Felix, et al., 2018).

Com relação ao domínio clareza dos objetivos, a média foi 6,43. Além disso, o presente estudo mostrou que quanto maior o período acadêmico dos jogadores mais claro o objetivo do jogo se tornava, ou seja, isso mostra que quanto mais o alunos avançam nos períodos escolares, mais claros vão ficando os objetivos das disciplinas e, por consequência, isso se reflete no objetivo do jogo que tem como finalidade promover a aprendizagem do exame físico dos doze pares de nervos cranianos. Tsuda, et al., (2014), ao avaliar o jogo "Armas e barões", também obtiveram a nota seis, referindo que, na questão narrativa e educativa, o jogador pode não notar que o jogo se trata de uma adaptação. Ramos, et al., (2018) mencionam que os jogos propõem objetivos, limitam ações pela normas e fornecem desafios que envolvem a habilidade de resolução de problemas. Nesse caso, o jogo NeuroGame-Card se propõe a desenvolver o pensamento clínico por meio dos desafios e da resolução de questões reflexivas.

No que tange ao feedback, quanto maior a pontuação maior a afinidade por jogos em grupo. É importante ressaltar que o jogo NeuroGame-Card busca fornecer um feedback constante do progresso da equipe no jogo, ao informar imediatamente se os jogadores erraram ou acertaram a pergunta feita, entretanto, no caso de erro, não é fornecida a resposta correta, o que pode deixá-los ansiosos em relação à afirmativa verdadeira. Tsuda, et al., (2014) apontam, em seu estudo, que os jogadores, ao finalizarem a fase com sucesso, são informados sobre as conquistas que alcançaram e, caso percam todos os pontos, é mostrada uma tela de "Game Over", informando que falharam. Ramos, et al., (2018) apontam que os jogos proporcionam estímulos, cores e movimentos que capturam facilmente os jogadores, especialmente aqueles que envolvem as tecnologias digitais. Nesse contexto, os jogadores recebem feedbacks constantes e imediatos, o que pode fortalecer a impaciência e o imediatismo demonstrado na atualidade.

Com referência ao domínio imersão, a literatura científica menciona que a imersão no jogo tem como finalidade conduzir o jogador para dentro do jogo, fazendo com que ele participe da história e sinta emoções que não estão vinculadas à vida real. Dessa forma, o competidor aperfeiçoa a própria interação social, tornando o jogo cooperativo e competitivo (Neves, et al., 2014). O jogo NeuroGame-Card, por envolver um trabalho em equipe, no qual esta tinha que acertar a alternativa correta ou realizar a ação proposta pelo cartão lúdico a fim de avançar o número de casas previamente estabelecidas pelo lançamento do dado, vencendo a equipe que alcançasse primeiro a casa "chegada" ao final do circuito do tabuleiro, fez com que os estudantes de Enfermagem tivessem imersão no jogo, o que pode ser verificado pela nota atribuída pelos estudantes após o jogo.

Estudo refere que os jogos devem criar e sustentar oportunidades que permitam interação social, como competição, colaboração e ligação entre os jogadores (Neves, et al., 2014). No domínio interação social, o presente estudo teve uma média de 5,79, provavelmente por se tratar de um jogo de equipe, no qual os membros tinham que se unir para responder às questões, o que fez com que houvesse interação social entre os jogadores. Os benefícios trazidos por essa interação social em sala de aula, com um objetivo comum, desenvolve fatores biológicos, como a capacidade cognitiva de raciocínio, a memória, a linguagem, a percepção, dentre outros (Mahoney \& Almeida, 2005).

Quanto ao domínio melhoria do conhecimento, a média foi 6,5, ou seja, alcançou quase a nota máxima que é sete. Percebe-se que os jogadores do NeuroGame-Card consideraram que o jogo melhorou consideravelmente o conhecimento. $\mathrm{O}$ 
resultado obtido nesse domínio coincide com a avaliação do jogo "Ortotetris" que obteve nota seis no mesmo domínio, justificando tal achado, em decorrência do aumento do conhecimento durante todo o jogo, pois, mesmo errando, o jogador percebe que a grafia que considerou a princípio está incorreta. Nesse caso, o conhecimento do participante, quanto à grafia de determinadas palavras, evolui (Tsuda, et al., 2014). Nesse sentido, o NeuroGame-Card também permitiu que ocorresse uma melhoria do conhecimento, pois, ao acertar ou errar as questões, o estudante passava a refletir sobre os conteúdos ministrados na disciplina com relação ao exame físico dos doze pares de nervos cranianos. A afinidade por jogos de estratégia, de tabuleiro e por jogos em grupo também afeta positivamente o domínio talvez por fortalecer o raciocínio clinico, uma vez que o grupo precisa traçar um caminho para certar as questões, fortalecendo a busca ou a discussão dos conhecimentos acerca dos doze pares de nervos cranianos.

Quanto à correlação de Spermam entre os domínios, a escala EGameFlow e as variáveis do perfil e de afinidade por jogos, houve correlação negativa entre o domínio clareza dos objetivos e afinidade por jogos. Esse resultado pode ser em decorrência de os alunos que possuem uma afinidade grande por jogo também apresentarem um amplo conhecimento sobre jogos, consequentemente apresentam uma base mais ampla para comparação, sendo este, por sua vez, mais rígida. O mesmo foi encontrado no estudo "Aprender com jogos e situações problema" no qual a participação ativa do jogador sobre o conhecimento próprio é dado por dois motivos: o primeiro deles deve-se ao fato de oferecer uma oportunidade para o jogador construir uma relação harmoniosa para a absorção de conhecimento, mostrando, assim, que o ato de aprender pode ser uma experiência desafiadora e interessante. O segundo motivo é valorizar o desenvolvimento do jogador sobre o próprio raciocínio, construindo o saber crítico sobre as questões levantadas do jogo, fazendo com que o sujeito crie estratégias para melhorar o desempenho (Macedo, 2007).

Outro aspecto importante foi o efeito positivo de ser do sexo feminino nos domínios autonomia, imersão e melhoria do conhecimento. Eventualmente, por apresentam maioria nos grupos, as mulheres exerceram um papel mais ativo na condução da partida e obtiveram maior interesse e rendimento. Seria interessante aumentar a participação de homens em proporção igual ou representativa e avaliar se este efeito continua. Os jogos podem carregar por si só vieses de gênero e estes precisam ser avaliados e evitados (Fontoura, et al. 2019), principalmente em se tratando de um curso predominantemente composto de estudantes mulheres.

Os achados deste estudo sobre as relações preditivas entre o perfil e a afinidade por determinado tipo de jogo na capacidade de predizer os domínios do EGameFlow são limitados e não mostraram um padrão claro, mas mostram que estudos nessa linha podem auxiliar na construção e na validação de jogos aplicados a ensino-aprendizagem em qualquer área. (Veja a nova redação da frase anterior representa a informação pretendida.) Apesar disso, ficou claro que os domínios do EGameFlow são afetados diferentemente pela afinidade dos jogadores por determinado tipo de jogo ou até pelo perfil. Por exemplo, o aumento da idade diminuiu o domínio desafio. Isso é importante, uma vez que o EGameFlow tem sido mostrado como eficiente para predizer o incremento de conhecimento após a aplicação de jogos (Ebrahimzadeh \& Alavi 2016; Espinosa-Curiel et al. 2020). As variáveis do perfil e a construção de escalas validadas para a detecção de afinidade por determinado tipo de jogo poderiam ser muito úteis para delimitar o interesse de grupos-alvo e orientar o tipo de jogo a ser elaborado, o que poderia levar a melhores resultados no processo ensino-aprendizagem independentemente da área.

\section{Conclusão}

A utilização do jogo NeuroGame-Card como estratégia educacional, para o ensino do exame físico dos doze pares de nervos cranianos, foi considerada uma experiência satisfatória por ter obtido uma média de 6,43 no domínio clareza dos objetivos e, no domínio melhoria do conhecimento, a média foi 6,50 , com os demais domínios maior ou igual a 5,19. No que tange a afinidade 
por jogos individuais ou em grupo e sua afinidade por determinado tipo de jogo foram capazes de predizer os domínios do EGameFlow.

Dessa forma, os estudantes puderam melhorar os conhecimentos de forma descontraída, motivando a participação e a interação de todos no processo de ensino e de aprendizagem. Destaca-se que grande parte dos estudantes de Enfermagem que participaram do jogo considerou que este melhora o conhecimento.

Como limitações deste trabalho, pode-se aprontar o tamanho amostral e sua especificidade, a pouca representatividade do sexo masculino, a aplicação em um único curso de graduação, entretanto, futuras pesquisas científicas contribuirão para consolidar e reforçar a utilização do jogo NeuroGame-Card em outros cursos de graduação da área da saúde e com maior número de participantes.

Ressalta-se que a utilização do jogo no ensino de Enfermagem representa um componente importante que facilita as atividades reflexivas e interativas que são fundamentais para a atividade do profissional de Enfermagem.

\section{Referências}

Castro, M. J., López, M., Cao, M. J., Fernández-Castro, M., García, S., Frutos, M., \& Jiménez, J. M. (2019). Impact of educational games on academic outcomes of students in the Degree in Nursing. PloS one, 14(7), e0220388.

Dias, J. D., Mekaro, M. S., Cheng Lu, J. K., Otsuka, J. L., Fonseca, L. M. M., \& Zem-Mascarenhas, S. H. (2016). Serious game development as a strategy for health promotion and tackling childhood obesity. Revista latino-americana de enfermagem, 24.

Santos, K. R., de Lemos, M. P. F., Araújo, H. O., de Oliveira, J., de Sousa Júnior, S. C., \& Fonseca, B. M. D. S. M. (2020). Jogo lúdico e educativo como ferramenta de ensino e aprendizagem em parasitologia. Revista Brasileira de Educação e Saúde, 10(1), 70-79.

Ebrahimzadeh, M., \& Alavi, S. (2016). Motivating EFL students: E-learning enjoyment as a predictor of vocabulary learning through digital video games. Cogent Education, 3(1), 1255400 .

Espinosa-Curiel, I.E., Pozas-Bogarin E.E., Martínez-Miranda J., Pérez-Espinosa H. (2020) Relationship Between Children's Enjoyment, User Experience Satisfaction, and Learning in a Serious Video Game for Nutrition Education: Empirical Pilot Study. JMIR Serious Games. 17; 8 (3): e21813.

Favaretto, D. V. (2017). Construção e aplicação de um jogo de tabuleiro para o ensino de física_(dissertação de mestrado), 52p.

Felix, Z. C., Machado, L. S., Silva, J. R. O., Silva, A. T. M. C., \& Almeida, L. R. (2018). Avaliando Satisfação do Usuário a Partir dos Modelos GameFlow e PENS: Um Estudo com o Jogo Caixa de Pandora Mobile. Proceedings of SBGames.

Fontoura, M. M, Rodrigues, L., Leite, P. S., Amaral, M. A., Almeida, L. D. A., Merkle, L. E. (2019) Relações de gênero em mecânicas de jogos. Proceedings of SBGames 2019, 794-803.

Fu, F. L., Su, R. C., \& Yu, S. C. (2009). EGameFlow: A scale to measure learners' enjoyment of e-learning games. Computers \& Education, 52(1), 101-112.

Gallegos C, Tesar AJ, Connor K, Martz K. (2017). The use of a game-based learning platform to engage nursing students: A descriptive, qualitative study. Nurse Educ Pract, 27,101-106.

Gómez-Urquiza, J. L., Gómez-Salgado, J., Albendín-García, L., Correa-Rodríguez, M., González-Jiménez, E., \& Cañadas-De la Fuente, G. A. (2019). The impact on nursing students' opinions and motivation of using a "Nursing Escape Room" as a teaching game: A descriptive study. Nurse education today, 72 , 73-76.

Hirsch, C. D., Barlem, E. L. D., Duarte, F. D., Fornari, N. C., Silva, B. R., \& Nogário, A. C. D. (2014). Sociodemographic and academic profile of undergraduate nursing student of the Nursing School/FURG. J Nurs Socioe Health, 1(1), 13-8.

Holanda, G. F., Andrade, L. Z. C., Freitas, D. T., da Silva, V. M., de Oliveira Lopes, M. V., \& de Araújo, T. L. (2012). Desenvolvimento e validação de jogo educativo: medida da pressão arterial. Revista Enfermagem UERJ, 20(3), 323-327.

Johnsen, H. M., Fossum, M., Vivekananda-Schmidt, P., Fruhling, A., \& Sletteb $\varnothing$, A. (2018). Developing a serious game for nurse education. Journal of gerontological nursing, 44(1), 15-19.

Macedo, L., Petty, A. L. S., \& Passos, N. C. (2007). Aprender com jogos e situações-problema [recurso eletrônico]. Lino de Macedo, Ana Lúcia Sícoli Petty, Norimar Christie Passos-Dados eletrônicos-Porto Alegre: Artmed.

Mahoney, A. A., \& de Almeida, L. R. (2005). Afetividade e processo ensino-aprendizagem: contribuições de Henri Wallon. Psicologia da Educação. Programa de Estudos Pós-Graduados em Educação: Psicologia da Educação. ISSN 2175-3520, (20).

Maddineshat, M., Yousefzadeh, M. R., Mohseni, M. A. H. D. I., Maghsoudi, Z., \& Ghaffari, M. E. (2018). Teaching ethics using games: Impact on Iranian nursing students' moral sensitivity. Indian journal of medical ethics, 4(1), 14-20. 
Research, Society and Development, v. 10, n. 7, e14510716368, 2021

(CC BY 4.0) | ISSN 2525-3409 | DOI: http://dx.doi.org/10.33448/rsd-v10i7.16368

Mitchell, G., Leonard, L., Carter, G., Santin, O., \& Brown Wilson, C. (2021). Evaluation of a 'serious game'on nursing student knowledge and uptake of influenza vaccination. PloS one, 16(1), e0245389.

Nasiri, M., Amirmohseni, L., Mofidi, A., Paim, C. P. P., Shamloo, M. B. B., \& Asadi, M. (2019). Educational games developed for students in perioperative nursing: A systematic review and appraisal of the evidence. Nurse education in practice, 37, 88-96.

Neves, D. E., de Oliveira Santos, L. G. N., Santana, R. C., \& Ishitani, L. (2014). Avaliação de jogos sérios casuais usando o método GameFlow. Revista Brasileira de Computação Aplicada, 6(1), 45-59.

Olympio, Paula Cristina de Andrade Pires, \& Alvim, Neide Aparecida Titonelli (2018). Jogo de tabuleiro: uma gerontotecnologia na clínica do cuidado de enfermagem. Revista Brasileira de Enfermagem, 71 (Supl. 2), 818-826. https://doi.org/10.1590/0034-7167-2017-0365

Oliveira, T. D. (2001). Amostragem não probabilística: adequação de situações para uso e limitações de amostras por conveniência, julgamento e quotas. Administração on line, 2(3), 01-10.

Oliveira, R. Á. M. D., \& Oliveira, K. L. D. (2007). Leitura e condições de estudo em universitários ingressantes. Psic: revista da Vetor Editora, 8(1), 51-59.

Oliveira, D. M. D. P., Pereira, C. U., \& Freitas, Z. M. D. P. (2016). Conhecimento do enfermeiro sobre avaliação neurológica do paciente com trauma cranioencefálico. Rev. enferm. UFPE on line, 4249-4254.

Panosso, M. G., Souza, S. R. D., \& Haydu, V. B. (2015). Características atribuídas a jogos educativos: uma interpretação AnalíticoComportamental. Psicologia Escolar e Educacional, 19(2), 233-242.

Ramos, D. K., Fronza, F. C. A. O., \& Cardoso, F. L. (2018). Jogos eletrônicos e funções executivas de universitários. Estudos de Psicologia (Campinas), 35(2), 217-228.

Rodrigues, R. S., Rodrigues, L. M. D. C., Mendes-Rodrigues, C., \& Silva, P.C.S. (2020). Elaboração de jogo de tabuleiro educativo para avaliação dos pares de nervos cranianos-"Neurogame-card". International Journal of Development Research, 10(6), 37146-37154.

Tyerman, J., Luctkar-Flude, M., \& Baker, C. (2021). Rapid Development of a COVID-19 Assessment and PPE Virtual Simulation Game. Clinical Simulation in Nursing.

Thornton Bacon, C., Trent, P., \& McCoy, TP (2018). Aprimorando o pensamento sistêmico para alunos de graduação em enfermagem usando a sexta à noite no pronto-socorro. Journal of Nursing Education, 57 (11), 687-689.

Tsuda, M., Sanches, V. M., Ferreira, T. G., Otsuka, J. L., \& Beder, D. M. (2014). Análise de métodos de avaliação de jogos educacionais. Proceedings of XIII SBGames, 12-14. 